\title{
Estrogen receptor coregulator binding modulator (ERX-11) enhances the activity of CDK4/6 inhibitors against estrogen receptor-positive breast cancers
}

Suryavathi Viswanadhapalli ${ }^{1 \dagger}$, Shihong Ma ${ }^{2 \dagger}$, Gangadhara Reddy Sareddy ${ }^{1,3}$, Tae-Kyung Lee ${ }^{4}$, Mengxing Li ${ }^{1}$,

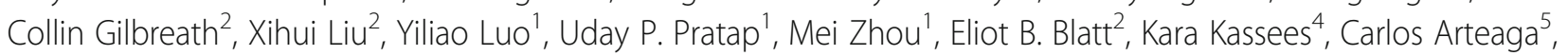
Prasanna Alluri ${ }^{6}$, Manjeet Rao ${ }^{7}$, Susan T. Weintraub ${ }^{8}$, Rajeshwar Rao Tekmal ${ }^{1}$, Jung-Mo Ahn ${ }^{4 *}$, Ganesh V. Raj ${ }^{2,5^{*}}$ and Ratna K. Vadlamudi ${ }^{1,3^{*}}$ (D)

\footnotetext{
Abstract

Background: CDK4/6 inhibitors in combination with endocrine therapy (AE/AI/SERDs) are approved for the treatment of ER+ advanced breast cancer (BCa). However, not all patients benefit from CDK4/6 inhibitors therapy. We previously reported a novel therapeutic agent, ERX-11, that binds to the estrogen receptor (ER) and modulates ER-coregulator interactions. Here, we tested if the combination of ERX-11 with agents approved for ER+ BCa would be more potent.

Methods: We tested the effect of combination therapy using BCa cell line models, including those that have acquired resistance to tamoxifen, letrozole, or CDK4/6 inhibitors or have been engineered to express mutant forms of the ER. In vitro activity was tested using Cell Titer-Glo, MTT, and apoptosis assays. Mechanistic studies were conducted using western blot, reporter gene assays, RT-qPCR, and mass spectrometry approaches. Xenograft, patient-derived explants (PDEs), and xenograft-derived explants (XDE) were used for preclinical evaluation and toxicity.

\footnotetext{
* Correspondence: jungmo.ahn@utdallas.edu;

ganesh.raj@utsouthwestern.edu; vadlamudi@uthscsa.edu

†Suryavathi Viswanadhapalli and Shihong Ma have equal contribution and must be considered as joint first authors.

${ }^{4}$ Department of Chemistry and Biochemistry, University of Texas at Dallas, Richardson, TX 75080, USA

${ }^{2}$ Departments of Urology and Pharmacology, University of Texas

Southwestern Medical Center at Dallas, Dallas, TX 75390, USA

'Department of Obstetrics and Gynecology, University of Texas Health, San

Antonio, TX 78229, USA

Full list of author information is available at the end of the article
}

(c) The Author(s). 2019 Open Access This article is distributed under the terms of the Creative Commons Attribution 4.0 International License (http://creativecommons.org/licenses/by/4.0/), which permits unrestricted use, distribution, and reproduction in any medium, provided you give appropriate credit to the original author(s) and the source, provide a link to the Creative Commons license, and indicate if changes were made. The Creative Commons Public Domain Dedication waiver (http://creativecommons.org/publicdomain/zero/1.0/) applies to the data made available in this article, unless otherwise stated. 
(Continued from previous page)

Results: ERX-11 inhibited the proliferation of therapy-resistant BCa cells in a dose-dependent manner, including ribociclib resistance. The combination of ERX-11 and CDK4/6 inhibitor was synergistic in decreasing the proliferation of both endocrine therapy-sensitive and endocrine therapy-resistant BCa cells, in vitro, in xenograft models in vivo, xenograft-derived explants ex vivo, and in primary patient-derived explants ex vivo. Importantly, the combination caused xenograft tumor regression in vivo. Unbiased global mass spectrometry studies demonstrated profound decreases in proliferation markers with combination therapy and indicated global proteomic changes in E2F1, ER, and ER coregulators. Mechanistically, the combination of ERX-11 and CDK4/6 inhibitor decreased the interaction between ER and its coregulators, as evidenced by immunoprecipitation followed by mass spectrometry studies. Biochemical studies confirmed that the combination therapy significantly altered the expression of proteins involved in E2F1 and ER signaling, and this is primarily driven by a transcriptional shift, as noted in gene expression studies.

Conclusions: Our results suggest that ERX-11 inhibited the proliferation of BCa cells resistant to both endocrine therapy and CDK4/6 inhibitors in a dose-dependent manner and that the combination of ERX-11 with a CDK4/6 inhibitor may represent a viable therapeutic approach.

Keywords: Estrogen receptor (ER), ER coregulators, Breast cancer, ER coregulator modulator, Therapy-resistant breast cancer, CDK4/6 inhibitor, Palbociclib

\section{Introduction}

Breast cancer $(\mathrm{BCa})$ is the most common cancer in women. The majority of $\mathrm{BCa}(70 \%)$ is estrogen receptorpositive $(\mathrm{ER}+)$. ER is activated by estrogenic ligands like estradiol (E2). ER signaling plays a key role in $\mathrm{BCa}$ cell cycle progression from $G_{1}$ to $S$ phase and is a critical molecular driver of $\mathrm{BCa}$ tumorigenesis. The primary therapeutic options for patients with systemic ER+ BCa are drugs targeting ER signaling, using either competitive antagonists like antiestrogens (AE) or antiestrogens like aromatase inhibitors (AI). However, most patients develop resistance to these endocrine drugs, and disease recurrence and progression is common [1, 2]. Importantly, the majority of endocrine therapy-resistant tumors retain ER signaling, through either mutation in the ER, alternative ligands, or altered coregulator profiles.

ER signaling requires ER interaction with oncogenic coregulator proteins $[3,4]$. Over one third $(38 \%)$ of ER coregulators are over-expressed in $\mathrm{BCa}$ [4-6] including oncogenic coregulators SRC3 [7, 8], SRC2 [9], and PELP1 [10]. These deregulated coregulators contribute to mammary tumorigenesis [6], therapy resistance, and metastases [11-14]. Alterations in the concentration or activity of oncogenic coregulators enable ER signaling from AE-ER complexes, effectively converting the antagonist to an agonist $[15,16]$. Recent studies also showed ER mutations lead to constitutive activity by enhancing ER-coregulator interactions with reduced sensitivity to ER antagonists, and mutations such as Y537S contribute to fulvestrant resistance in vivo [17].

Transcriptional and post-translational regulation of ER coregulators [18] may play a critical role in ER regulation of cell cycle progression. The activity of cyclindependent kinases (CDKs) [19] is known to regulate several coregulators via phosphorylation. Luminal breast cancer commonly exhibits cyclin D1 and CDK4 amplification [20], while endocrine therapy-resistant tumors often exhibit deregulation of the CDK4/6 pathway [21]. Recently, the combination of CDK4/6 inhibitors and endocrine therapy (AE/AI/SERDs) has been approved for the treatment of ER+ advanced $\mathrm{BCa}$ [22]. Three selective CDK4/6 inhibitors (palbociclib, ribociclib, and abemaciclib) bind to the ATP-binding pocket of CDK4/6 and produce a cytostatic effect in combination with agents that target ER signaling [21]. However, some patients do not benefit from this combination, and the emergence of resistance in responders is common.

We recently reported the development of a small organic molecule, ERX-11 [23] (ER coregulator binding modulator-11), that uniquely interacts with ER and blocks the interaction of selective oncogenic coregulators with ER. ERX-11 was shown to block ER signaling and ERdriven proliferation in therapy-sensitive and therapyresistant ER+ BCa. Since endocrine therapy-resistant tumors retain functional ER signaling via oncogenic coregulator proteins, we reasoned that the combination of ERX11 with CDK4/6 inhibitors could be synergistic and prevent the emergence of resistant phenotypes.

In this manuscript, we tested and demonstrated the utility of the combination of ERX-11 and CDK4/6 inhibitors (palbociclib, abemaciclib, ribociclib) in treating therapy-sensitive and therapy-resistant advanced $\mathrm{BCa}$. These studies provide the preclinical rationale for this combination therapy.

\section{Materials and methods}

Cell lines

Human BCa cells MCF-7, ZR-75, and T-47D were obtained from the American Type Culture Collection 
(ATCC, Manassas, VA). ZR-75-ESR1-MT-D538G and ZR-75-ESR1-MT-Y537S cells were described earlier and were cultured in RPMI media containing $10 \%$ fetal bovine serum (FBS) [23]. Ribociclib-resistant cells (MCF-7/RR) were described earlier [24]. MCF-7/ TamR cells [25] and MCF-7/LTLTca cells [26] were described earlier. MCF-7/LTLTca and MCF-7/TamR cells were cultured in phenol red-free RPMI medium containing 5\% dextran-coated charcoal-treated FBS (DCC-FBS) supplemented with either $1 \mu \mathrm{mol} / \mathrm{L}$ of letrozole or $1 \mu \mathrm{mol} / \mathrm{L}$ of tamoxifen, respectively. All the model cells utilized are free of mycoplasma contamination. Additionally, STR DNA profiling of the cells was used to confirm the identity using UTHSA and UT Southwestern core facilities.

\section{Reagents}

Letrozole, 17- $\beta$-estradiol, (Z)-4-hydroxytamoxifen, and androstenedione were purchased from Sigma (St. Louis, MO). Palbociclib was purchased from Cayman Chemical (MI, USA). Ribociclib (HY-15777) and abemaciclib (HY16297A) were purchased from MedChem Express LLC (Monmouth Junction, NJ). Ki-67 anti-human clone MIB-1 antibody (cat\#M7240) was purchased from Dako (Carpinteria, CA). The PELP1, SRC3, and SRC1 antibodies were purchased from Bethyl Laboratories (Montgomery, TX). The GAPDH, p-ERK1/2, ERK1/2, p-4EBP1, 4EBP1, pmTOR(S2448), mTOR, p-p70S6K(T389), p70S6K, p21, PARP, and p65 antibodies were purchased from Cell Signaling Technology (Beverly, MA). The E2F1 and FOXM1 antibodies were purchased from Santa Cruz Biotechnology. The anti-ER alpha and anti-phospho-histone H2A.X (Ser139) antibodies were purchased from Millipore. The cyclin D1, $\beta$-actin, and all secondary antibodies were purchased from Sigma. ERX-11 was synthesized by following the previously reported procedure [23].

\section{Cell viability and colony formation assays}

The effects of ERX-11 and CDK4/6 inhibitors on cell viability were measured using the MTT cell viability assay in 96-well plates. BCa cells were seeded in 96-well plates $\left(1 \times 10^{3}\right.$ cells/well $)$ in phenol red-free RPMI medium containing 5\% DCC-FBS. After an overnight incubation, cells were treated with varying concentrations of the ERX-11, palbociclib, abemaciclib, ribociclib, or combination of ERX-11 with each of CDK4/6 inhibitors in the presence or absence of estrogen $(\mathrm{E} 2)\left(10^{-8} \mathrm{M}\right)$ for 7 days. For some experiments, cell viability was also measured using Cell Titer-Glo luminescent cell viability assay (Promega) in 96-well, flat, clear-bottom, opaquewall microplates according to the manufacturer's protocol. For colony formation assays, MCF-7, ZR-75, ZR-75ESR1-MT-Y537S, and ZR-75-ESR1-MT-D538G model cells (500 cells/well) were seeded in 6-well plates, treated with indicated drugs, and allowed to grow for 14 days. The cells were fixed in ice-cold methanol and stained with $0.5 \%$ crystal violet solution. Pixels above a threshold of 5000 in their intensity value were counted.

\section{Western blotting and immunoprecipitation}

Western blotting and immunoprecipitation were performed as described previously [23]. Briefly, for western blot, whole-cell lysates were prepared using RIPA buffer containing protease and phosphatase inhibitors (Sigma Chemical Co.). Total proteins $(50 \mu \mathrm{g})$ were mixed with SDS sample buffer and subjected to SDS-PAGE. Blots were developed using the ECL kit (Thermo Scientific, Waltham, MA).

\section{Cell cycle analysis}

MCF-7 and MCF-7/TamR cells were seeded in 100-mm culture plates, and after overnight incubation, cells were treated with either vehicle, ERX-11 $(1 \mu \mathrm{M})$, palbociclib $(250 \mathrm{nM})$, or combination in the presence of estrogen (E2) $\left(10^{-8} \mathrm{M}\right)$ for $48 \mathrm{~h}$. Cells were then trypsinized and harvested in PBS, followed by fixation in ice-cold $70 \%$ ethanol for $30 \mathrm{~min}$ at $4{ }^{\circ} \mathrm{C}$. Cells were washed again with PBS and stained with a mixture of $50 \mu \mathrm{g} / \mathrm{mL}$ propidium iodide and $50 \mu \mathrm{g} / \mathrm{mL}$ RNase A. The PI-stained cells were subjected to flow cytometry using a FACS Calibur (BD Biosciences).

\section{Animal studies}

All animal experiments were performed after obtaining UTHSA IACUC approval using methods in the approved protocol. For xenograft studies, $2 \times 10^{6} \mathrm{MCF}-7 /$ TamR and MCF-7/LTLT model cells were mixed with an equal volume of Matrigel and implanted in the mammary fat pads of 6-week-old female nude mice implanted with tamoxifen and androstenedione pellets, respectively as described [27]. When the tumor was established, it was dissected into small pieces and they were again implanted subcutaneously into the nude mice. Once the tumors reached a measurable size, mice were randomly selected to receive vehicle and treatment with ERX-11 $(10 \mathrm{mg} / \mathrm{kg} /$ day orally), palbociclib $(50 \mathrm{mg} / \mathrm{kg} /$ day orally), or in combination ( $n=4-7$ tumors). Tumor growth was measured with a caliper at 3-4-day intervals. At the end of each experiment, the mice were euthanized, and the tumors were removed, weighed, and processed for immunohistochemical staining.

\section{Xenograft-derived explant and patient-derived explant studies}

For xenograft-derived explant (XDE) studies, xenograft tumors of ZR-75-ESR1-MT-D538G, ZR-75-ESR1-MTY537S, and MCF-7/LTLT were initially established in SCID mice as described above, and earlier published 
patient-derived explant (PDE) protocol [23] was adapted for XDE assay. When the tumor reached $700 \mathrm{~mm}^{3}$, they were dissected into 2-mm cubes. Tumor samples were incubated on gelatin sponges for $24 \mathrm{~h}$ in culture medium containing 10\% FBS, followed by treatment with either vehicle, $1 \mu \mathrm{M}$ ERX-11, $500 \mathrm{nM}$ palbociclib, or combination for $72 \mathrm{~h}$. For PDE studies, excised tissue samples were processed and cultured ex vivo as previously described [23]. De-identified patient tumors were obtained from the UTSW Tissue Repository after institutional review board approval (STU-032011-187, 28). Briefly, tumor samples were incubated on gelatin sponges for $24 \mathrm{~h}$ in culture medium containing $10 \% \mathrm{FBS}$, followed by treatment with either vehicle, $10 \mu \mathrm{M}$ ERX-11, $250 \mathrm{nM}$ palbociclib, or combination for $72 \mathrm{~h}$. Representative tissues were fixed in $10 \%$ formalin at $4{ }^{\circ} \mathrm{C}$ overnight and subsequently processed into paraffin blocks. The sections were then processed for immunohistochemical analysis.

\section{RT-qPCR}

Reverse transcription (RT) reactions were performed by using SuperScript III First Strand kit (Invitrogen, Carlsbad), according to the manufacturer's protocol. Realtime PCR was done using SYBR Green on an Illumina Real-Time PCR system. Primer sequences of the genes used were obtained from Harvard Primer Bank (https:// pga.mgh.harvard.edu/primerbank).

\section{Mass spectrometry-based DIA analyses of whole-cell lysates}

MCF-7/TamR cells were treated with E2 $\left(10^{-8} \mathrm{M}\right)$ plus vehicle or ERX-11 $(1 \mu \mathrm{M})$, palbociclib $(250 \mathrm{nM})$, or the combination of ERX-11 and palbociclib. After $72 \mathrm{~h}$ of the treatment, cells were pelleted and snap-frozen and then lysed 5\% SDS/50 mM TEAC (DIA). Protein concentrations were determined by EZQ Protein Quantitation kit (Thermo Fisher) and peptides by Pierce Quantitative Fluorometric Peptide Assay (Thermo Fisher). For DIA analyses, S-Traps (Protifi) were used for reduction/alkylation, tryptic digestion, and cleanup, starting with $100 \mu \mathrm{g}$ of protein (yield $66.5 \pm 7.5 \mu \mathrm{g}$ ). A pool was made of the 12 samples (3 replicates from each group), and $2 \mu \mathrm{g}$ peptide aliquots were analyzed on the Lumos using gas-phase fractionation and 4- $m / z$ windows (120k resolution for precursor scans, $15 \mathrm{k}$ for product ion scans, all in the orbitrap) to produce a DIA chromatogram spectral library which was searched against the UniProt_human database. Experimental samples were blocked by replicate and randomized within each replicate. Injections of $2 \mu \mathrm{g}$ of peptides and a 2-h HPLC gradient were employed. MS data were acquired using $12-\mathrm{m} / z$ windows (staggered; $120 \mathrm{k}$ resolution for precursor scans, 30k for product ion scans) and searched against the chromatogram library.
Scaffold DIA (v1.3.1; Proteome Software) was used for all DIA data processing. The pathway analysis was conducted with Reactome Pathway Database (https://reactome.org/) on differentially expressed proteins, focusing on the group that exhibited $\geq 1.5$-fold change comparing mono and combination therapies to vehicle.

\section{Immunohistochemistry and $\mathrm{\gamma H} 2 \mathrm{AX}$ analysis}

Immunohistochemical studies were performed as described previously [23]. For the immunohistochemical studies, tissue sections were blocked in 5\% normal goat serum followed by overnight incubation with Ki-67 (1: 100) primary antibody and subsequent secondary antibody incubation for $30 \mathrm{~min}$ at room temperature. Immunoreactivity was visualized by using the DAB substrate and counterstained with hematoxylin (Vector Lab, Inc.). Percent of Ki-67-positive proliferating cells was calculated in five randomly selected microscopic fields. For $\gamma \mathrm{H} 2 \mathrm{AX}$ analysis, MCF-7 cells were cultured on glass coverslips and treated with vehicle, ERX-11, palbociclib, or combination for $4 \mathrm{~h}$. Cells were fixed in $3.7 \%$ paraformaldehyde followed by permeabilization with $0.1 \%$ TritonX-100 for $10 \mathrm{~min}$. Cells were then stained with $\gamma \mathrm{H} 2 \mathrm{AX}$ antibody, and the fluorescence was analyzed by microscopy.

\section{Statistical analyses}

Statistical differences between the groups were analyzed with either a $t$ test or ANOVA as appropriate using GraphPad Prism 6 software. All the data represented in plots are shown as means \pm SE. A value of $p<0.05$ was considered statistically significant.

\section{Results}

ERX-11 and CDK4/6 inhibitors act synergistically to reduce the growth of therapy-sensitive and therapyresistant $\mathrm{BCa}$ cells

Our recent studies demonstrated that ERX-11 had antiproliferative activity on therapy-resistant $\mathrm{ER}+\mathrm{BCa}$ cells, although at $500 \mathrm{nM}$ concentrations [23]. Since therapyresistant $\mathrm{BCa}$ cells often exhibit deregulation of both CDK4/6 and ER coregulator-driven pathways, we hypothesized that the combination of ERX-11 and CDK4/6 inhibitor would more effectively block $\mathrm{BCa}$ cell proliferation. Colony formation assays visually demonstrated that the combination was more effective in reducing the colony formation of $\mathrm{BCa}$ cells compared to either ERX-11 or palbociclib monotherapy (Fig. 1a).

Therapy-sensitive (ZR-75, T-47D) and endocrine therapy-resistant (MCF-7/TamR, MCF-7/LTLT) BCa cells were treated with ERX-11 or the CDK4/6 inhibitor, palbociclib, or with the combination. MTT assays showed that combination therapy was more effective in reducing the cell viability compared to either ERX- 
11 or palbociclib alone (Fig. 1b-e). The combination index (CI) studies using the Chou-Talalay method [28] showed that the CI value was less than 1 in all the four $\mathrm{BCa}$ model cells tested and confirmed that the combination therapy was synergistic. In contrast, the combination of ERX-11 with other therapeutic agents like gemcitabine, cisplatin, or paclitaxel did not show any additive or synergistic effect (Additional file 1: Figure S1). To further test whether palbociclib enhances the efficacy of ERX-11 by lowering its IC50, we conducted a dose-response study with ERX-11 keeping the constant concentration of palbociclib. The results showed that palbociclib significantly enhanced the potency of ERX-11 and lowered the IC50 of ERX-11 in all five model cells (Additional file 1: Figure S2).

We then tested the utility of combination therapy using two additional CDK4/6 inhibitors currently in clinical trials/treatment including abemaciclib and ribociclib. The results from these studies showed that similar to palbociclib, both abemaciclib and ribociclib act synergistically with ERX-11 (Fig. 1f, g). We also confirmed the utility of
ERX-11 combination therapy using three different CDK4/ 6 inhibitors in colony formation assays. Results showed that the ERX-11+CDK4/6 inhibitor combination was more effective in reducing the colony formation of $\mathrm{BCa}$ cells compared to either ERX-11, palbociclib, abemaciclib, or ribociclib monotherapy (Additional file 1: Figure S3).

We next treated ER-mutant model cells (ZR-75-ESR1MT-Y537S, ZR-75-ESR1-MT-D538G) with ERX-11 or the CDK4/6 inhibitors, or with the combination. The results showed that the combination therapy has a synergistic effect in these ER-mutant model cells (Fig. 2a, b, c). We then tested whether ERX-11 has utility in treating MCF-7/RR cells that have acquired resistance to the CDK4/6 inhibitor, ribociclib [24]. We noted that both MCF-7 and MCF-7/RR were responsive to ERX-11. As expected, due to the acquired resistance of MCF-7/RR cells to ribociclib, the combination of ERX-11+ribociclib was not additive in MCF-7/RR cells; however, they are sensitive to ERX-11 therapy (Additional file 1: Figure S4A). Furthermore, MCF-7/RR exhibited resistance to antiestrogen fulvestrant (ICI); however, ERX-11 was able

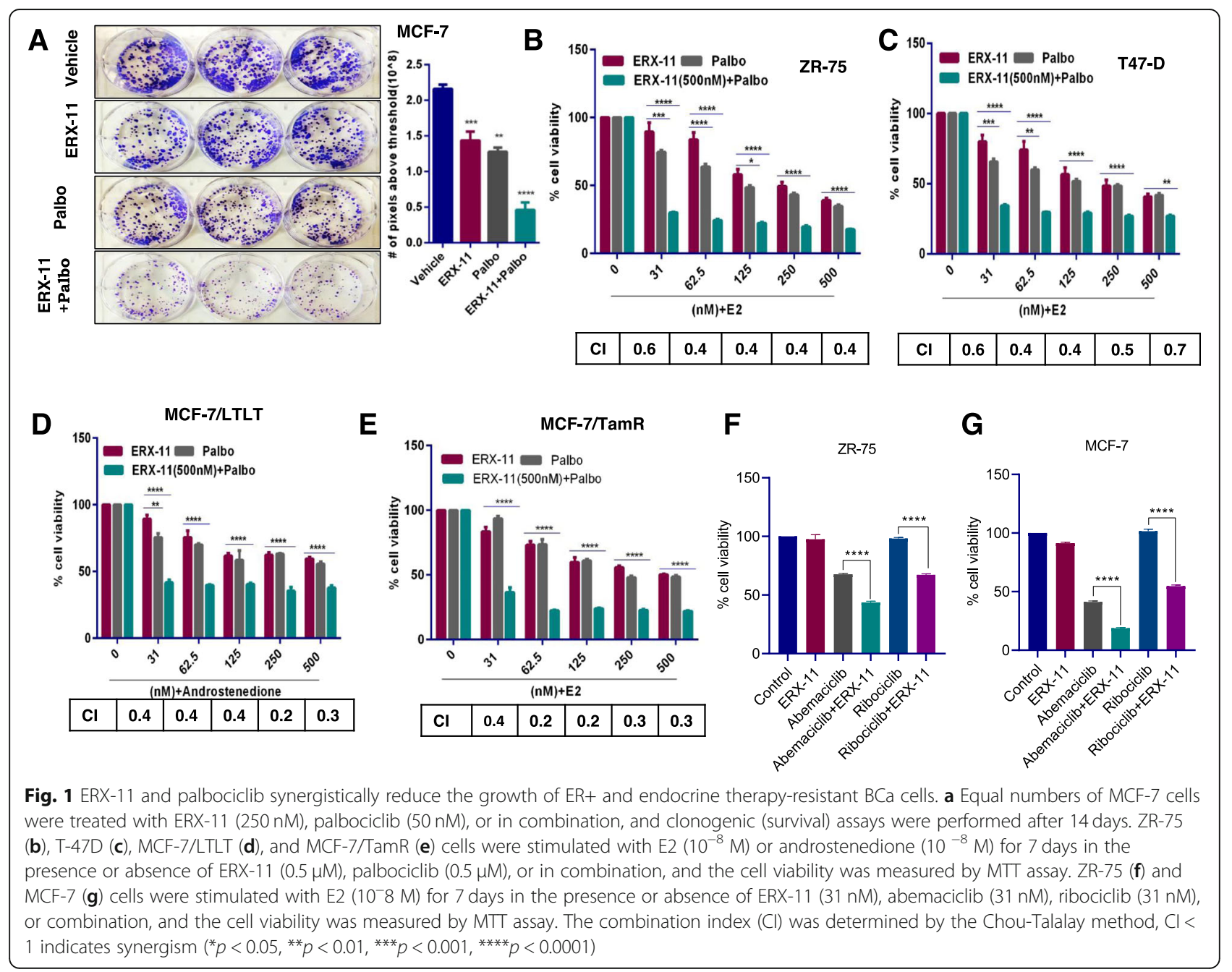


to reduce the cell viability of MCF-7/RR (Additional file 1: Figure S4B, C). Collectively, these results suggest that ERX-11-targeted molecular vulnerabilities enhance palbociclib efficacy, and combination therapy will have utility in treating therapy-sensitive and therapy-resistant BCa.

ERX-11 and CDK4/6 inhibitor combination is effective in reducing the proliferation of xenograft breast tumor explants

We then evaluated the effect of ERX-11 and palbociclib combination therapy using ex vivo cultures of xenograft tumors (xenograft-derived explants (XDE)) of two prevalent ER mutants (ZR-75-ESR1-MT-Y537S, ZR-75-ESR1MT-D538G) [29-32] and letrozole-resistant MCF-7/
LTLT tumors and examined the expression of Ki-67 as a marker of proliferation. The results showed that ERX-11 and palbociclib combination is more efficient in reducing the expression of Ki-67 in all three XDEs including those from MCF-7/LTLT, ZR-75-ESR1-MT-Y537S, and ESR1MT-D538G (Fig. 2d-g). These results validate our cell line data and suggest that the combination of ERX-11 and palbociclib can inhibit the growth of ER+ human breast tumors and therapy-resistant xenograft tumors ex vivo.

\section{Combination of ERX-11 with CDK4/6 inhibitor promoted tumor regression in preclinical xenograft models of endocrine resistance}

In vivo efficacy of ERX-11 and palbociclib combination therapy was evaluated using MCF-7/TamR

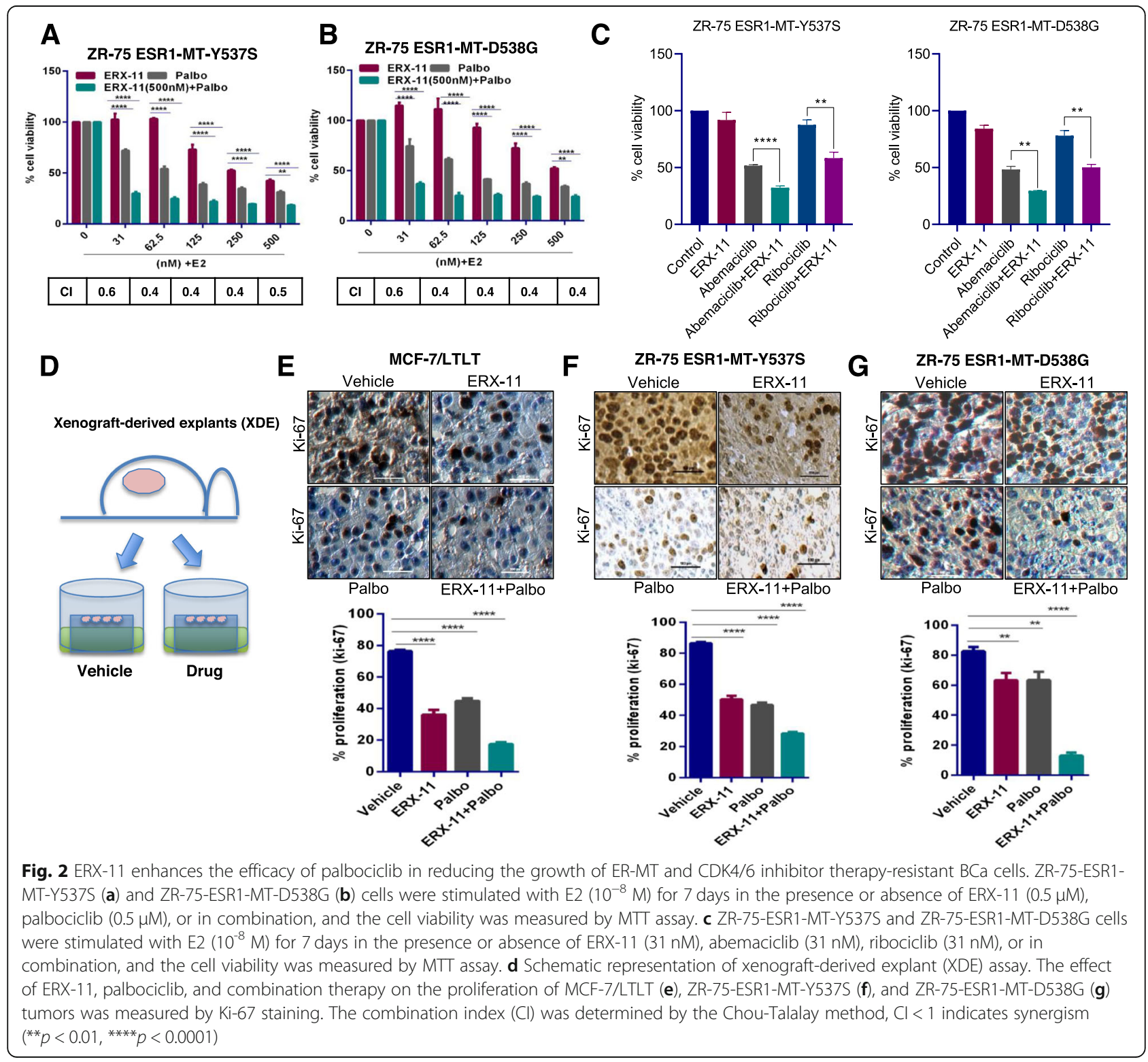


xenograft model that exhibits resistance to tamoxifen treatment. MCF-7/TamR xenografts $(n=4$ tumors/ group) containing nude mice were randomized to feed via oral gavage 5 days/week with $10 \mathrm{mg} / \mathrm{kg}$ ERX-11 or $50 \mathrm{mg} / \mathrm{kg}$ palbociclib or combination. Combination treatment resulted in significantly lower tumor volume and smaller tumor size compared to monotherapy of ERX-11 or palbociclib (Fig. 3a). Mice treated with ERX-11 and palbociclib combination exhibited no overt signs of toxicity, and body weight was not affected (Fig. 3b) compared to vehicle or monotherapy. Furthermore, ERX-11- and palbociclib-treated tumors exhibited less proliferation (Ki-67 staining) compared to vehicle or monotherapy (Fig. 3c, d). We also confirmed the efficacy of combination therapy using MCF-7/LTLT xenograft tumors that exhibit resistance to letrozole ( $n=7$ tumors/group). Results showed that ERX-11 and palbociclib combination is more efficient in reducing the letrozole-resistant tumor volume and proliferation (Fig. 3e-h). Collectively, these data indicate that ERX-11 and palbociclib combination is more potent in reducing the growth of endocrine therapyresistant breast tumors in vivo.

\section{ERX-11 and CDK4/6 inhibitor combination is effective in reducing the proliferation of primary patient-derived breast tumor explants}

To test the efficacy of ERX-11 and palbociclib combination therapy on primary breast specimens, we have used patient-derived explants (PDE) of primary breast tumors that allow for the evaluation of drugs on breast tumors while maintaining their native tissue architecture [33, 34]. Surgically extirpated deidentified breast tissues are sliced into small pieces and grown ex vivo on a gelatin sponge and treated with either vehicle, ERX-11, palbociclib, or ERX-

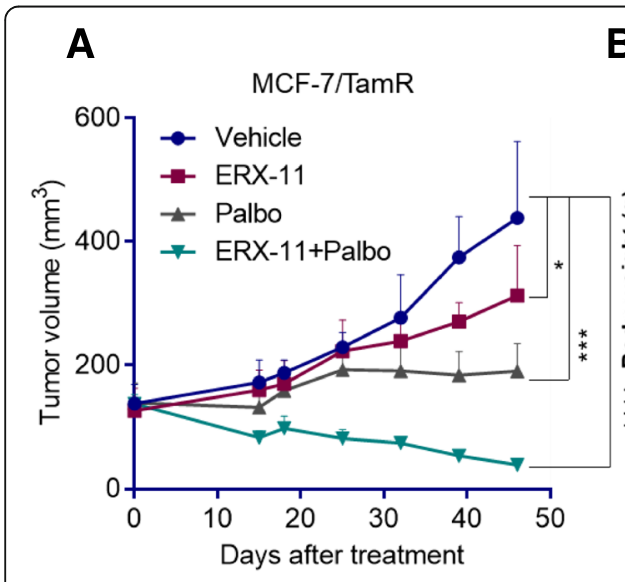

E

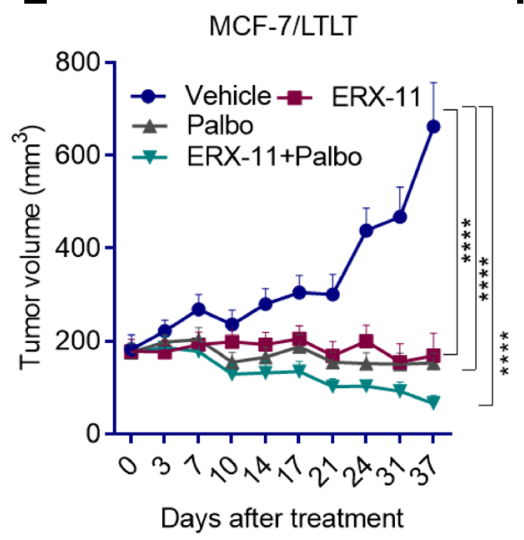

$\mathbf{F}$

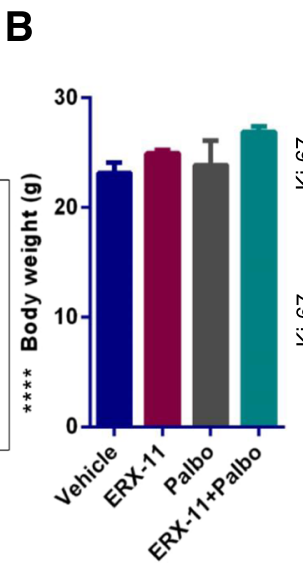

C
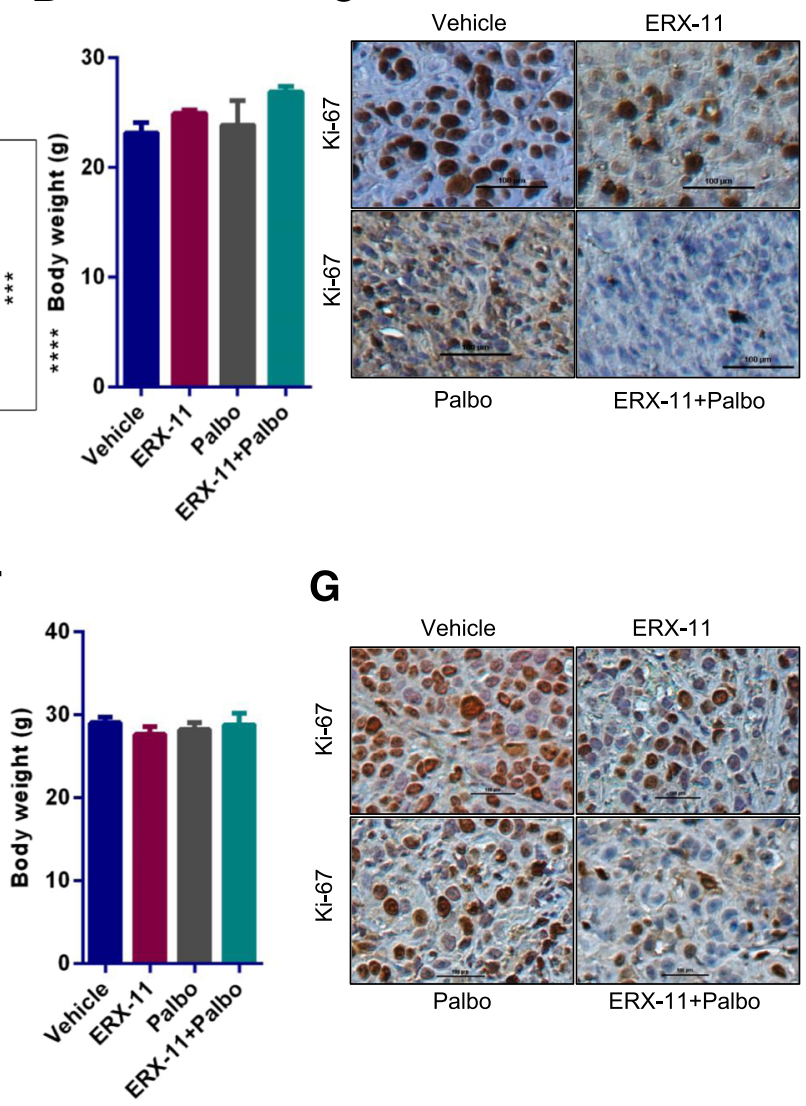

G

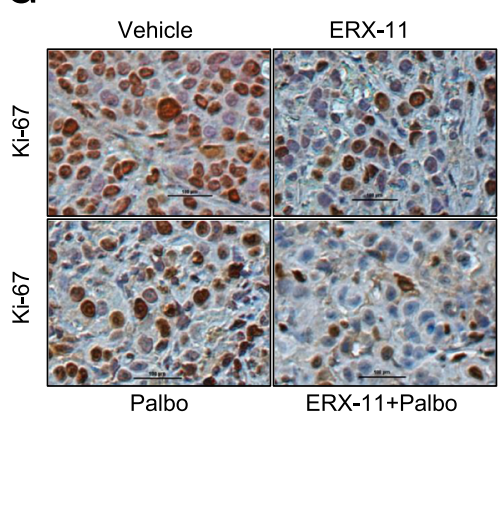

D

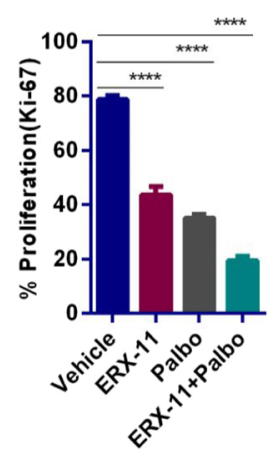

H

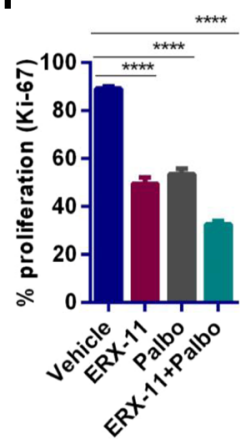

Fig. 3 ERX-11 enhances palbociclib ability to reduce tumor growth in preclinical models of endocrine therapy resistance. a-d Following implantation and growth of ER+ tamoxifen-resistant xenografts ( $n=4$ tumors/group), mice were treated with vehicle, ERX-11 (10 mg/kg/day), palbociclib (50 mg/kg/day), or in combination. Tumor volume (a), body weight (b), and Ki-67 status of vehicle and drug-treated tumors were shown $(\mathbf{c}, \mathbf{d})$. Following implantation and growth of ER+ letrozole resistant xenografts ( $n=7$ tumors/group), mice were treated with vehicle or ERX-11(10 mg/kg/day), palbociclib (50 mg/kg/day), or in combination. Tumor volume (e), body weight (f), and Ki-67 status of vehicle and drugtreated tumors were shown $(\mathbf{g}, \mathbf{h})\left({ }^{*} p<0.05,{ }^{* * *} p<0.001,{ }^{* * *} p<0.0001\right)$ 
11+palbociclib (Fig. 4a, b). Combination therapy of ERX-11 and palbociclib potently reduced the proliferation (Ki-67 staining) in PDEs obtained from 6/6 patients compared to untreated vehicle, ERX-11, or palbocliclib monotherapy (Fig. 4c, d).

\section{ERX-11 and CDK4/6 inhibitor treatment alters the} interactions between ER and coregulators

Since ERX-11 blocks ER interactions with coregulators, we examined the effect of the combination therapy on the interactions between ER with coregulators. Using an unbiased immunoprecipitation mass spectrometry approach, we showed that the combination therapy significantly disrupted the interactions between ER and a larger number of ERbinding coregulators than either monotherapy or vehicle controls. Indeed, the combination disrupted 136/199 coregulator interactions with ER in MCF-7 cells compared to 31 and 76 coregulators blocked by palbociclib or ERX-11 alone (Fig. 5a-d, Additional file 1: Figure S5). We also confirmed the ability of ERX-11+palbo combination therapy to potently disrupt ER $\alpha$ interaction with coregulators using an immunoprecipitation experiment that examined the interaction of two well-known ER $\alpha$ coregulators SRC1 and SRC3. The results confirmed that treatment of combination therapy potently disrupted the interaction of ER $\alpha$ with both SRC1 and SRC3 compared to monotherapy (Additional file 1: Figure S6A). Collectively, these studies suggested that coregulator interaction with ER was globally affected by the ERX-11 and palbociclib combination therapy.

\section{ERX-11 did not enhance CDK4/6 inhibitor-mediated G0- G1 cell cycle arrest}

Effects of ERX-11 and palbociclib combination therapy on cell cycle progression were investigated using cell

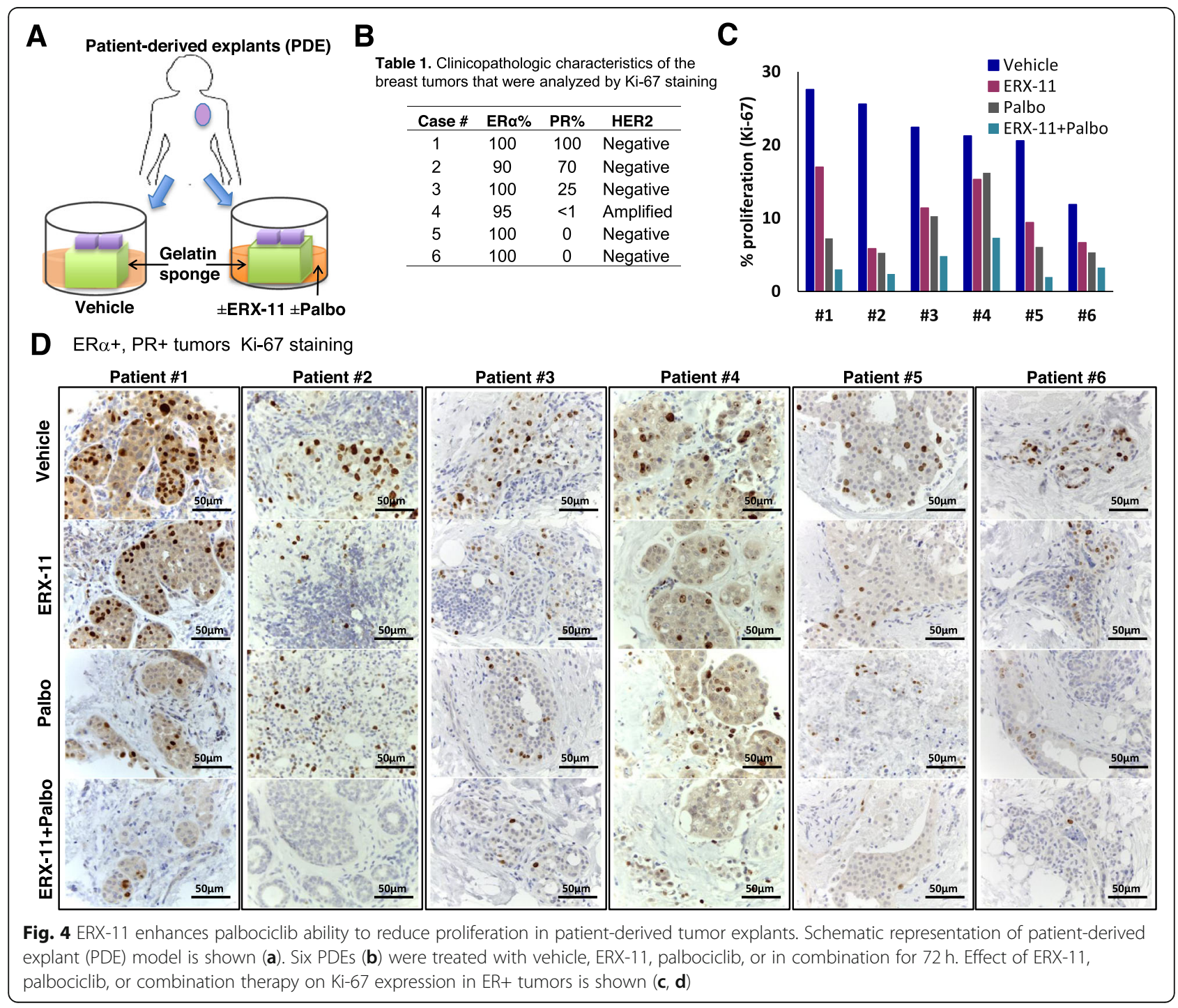




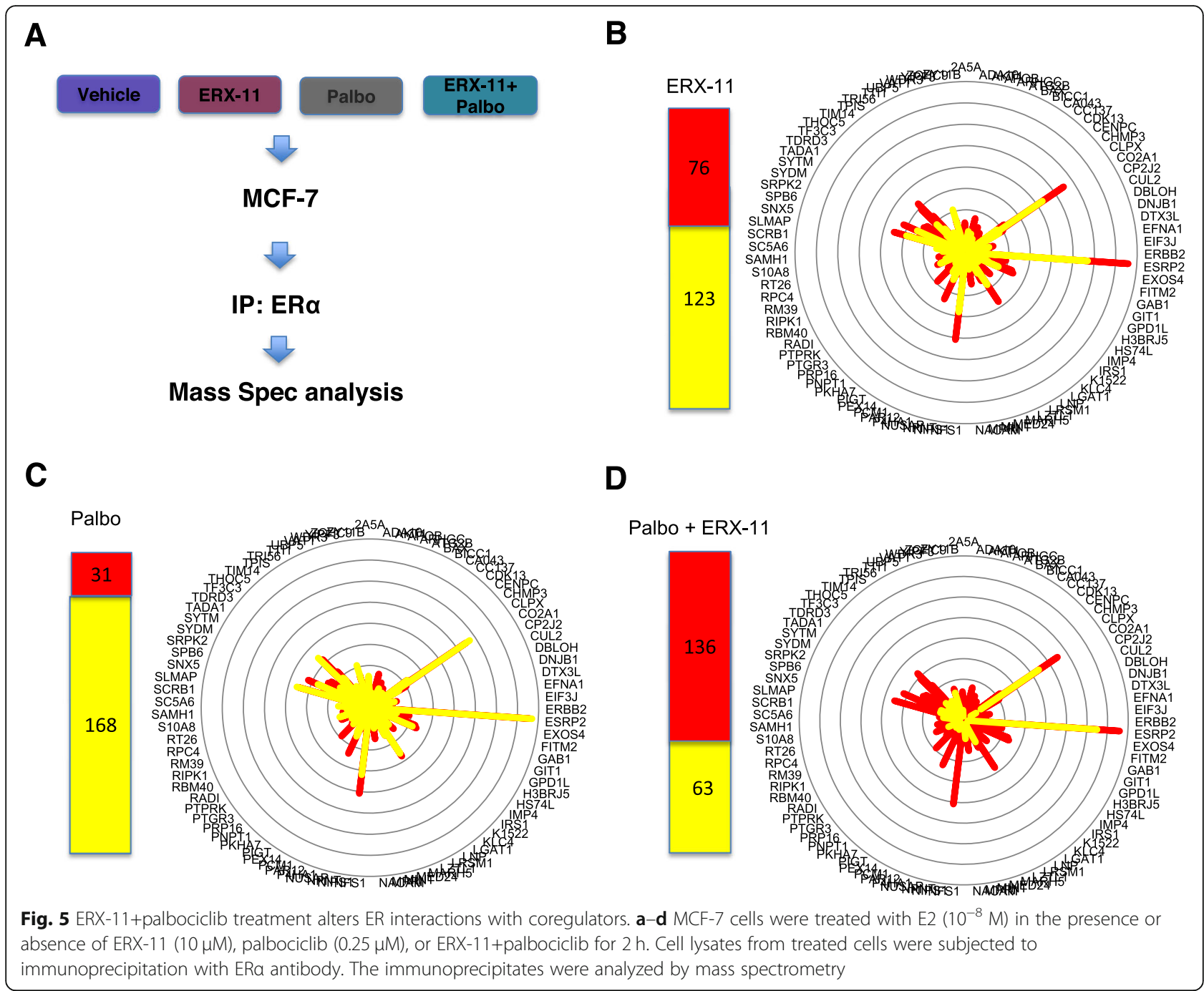

cycle analyses. As expected from the inhibition of CDK4/6 activity, both MCF-7 and MCF-7/TamR cells showed an increase in the G0-G1 phase of the cell cycle. However, combination therapy did not promote any further increase than palbociclib as a monotherapy (Additional file 1: Figure S6B). Western blot analysis also showed a lack of additive effect of combination therapy on the levels of various cell cycle proteins including cyclin D1, p21, and pERK1/2 (Additional file 1: Figure S6C). Collectively, these findings suggest that the higher efficacy seen in ERX-11 and palbociclib combination therapy is not due to cell cycle alterations.

\section{Global mass spectrometry-based analyses identified unique pathways modulated by combination therapy}

To understand the mechanism of synergy between ERX-11 and palbociclib, we used data-independent acquisition (DIA) mass spectrometry analyses using whole-cell lysates of MCF-7/TamR cells treated with vehicle, ERX-11, palbociclib, or combination of ERX-11 and palbociclib in 3 biological replicates. DIA analyses resulted in the identification of 4449 proteins (4442 quantified with 2 or more peptides). Proteins exhibiting $\geq 1.5$-fold difference (either up or down) in relative quantity after treatment with ERX-11, palbociclib, or combination of ERX-11+palbociclib compared to vehicle are shown in the Venn diagram (Fig. 6a). Importantly, in agreement with immunohistochemical results from PDE, XDE, and xenografts, DIA analysis showed significant downregulation of the proliferation marker Ki-67 in response to combination therapy compared to monotherapy or vehicle. In contrast, no effect on the expression of housekeeping genes such as GAPDH was observed by either mono or combination therapy (Fig. 6b). A comparison was made of relative protein abundance between vehicle and combination therapy, and proteins that were downregulated or upregulated $\geq 1.5$-fold were subjected to pathway analysis using Reactome (https://reactome.org/). Selected pathways are shown in Additional file 1: Figure S7. The unique 


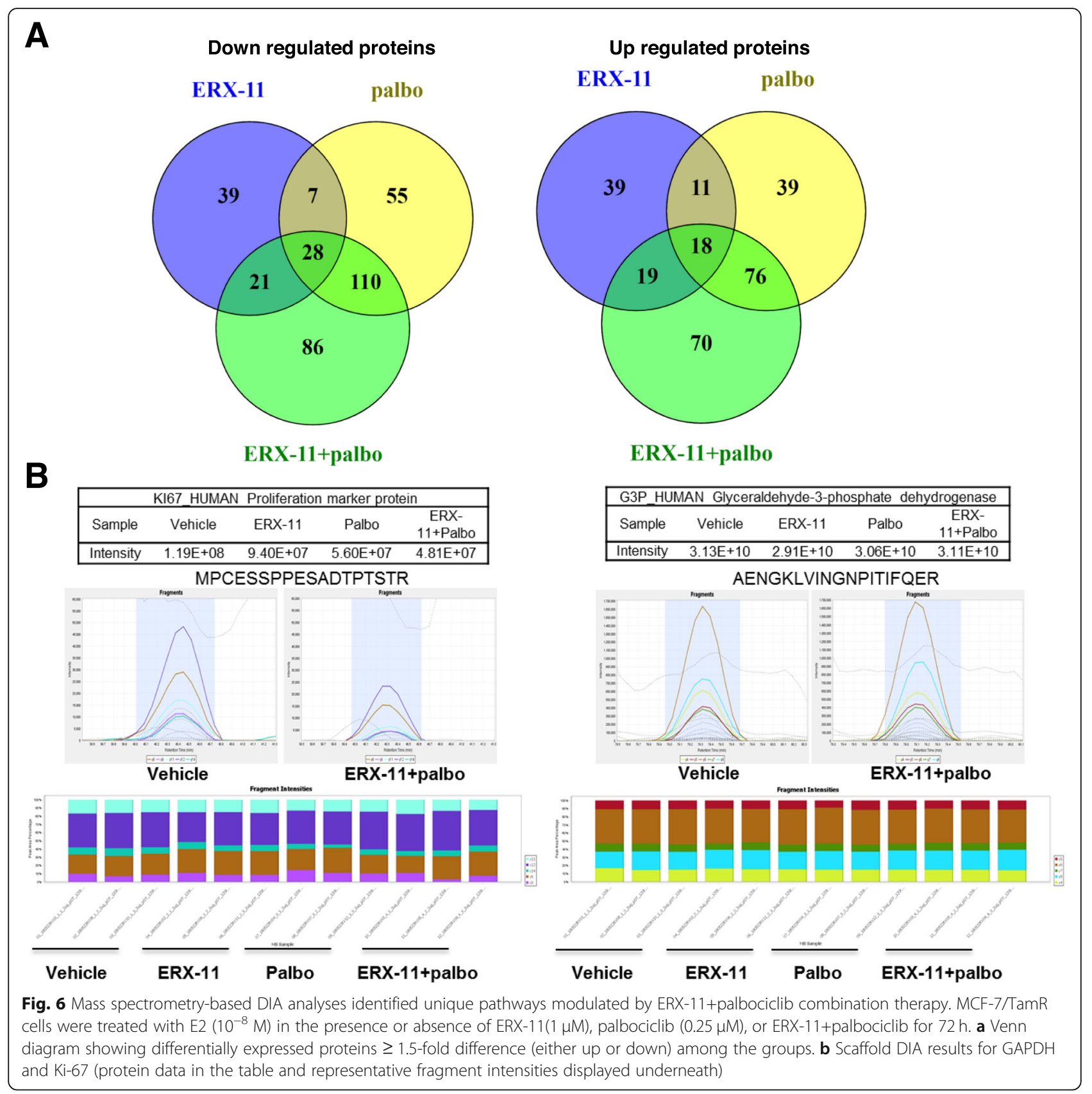

pathways modulated by the combination therapy include E2F signaling, RNA metabolism, translation, DNA damage, DNA repair, apoptosis, and endoplasmic reticulum stress. Representative results from the DIA analysis of selected proteins involved are shown (Additional file 1: Figure S8). We have utilized a widely used $\gamma-\mathrm{H} 2 \mathrm{AX}$ foci assay that indicates the presence of a double-strand break (DSB) and foci disappearance indicate repair of the DNA damage. The results confirmed an increase in the accumulation of $\gamma-\mathrm{H} 2 \mathrm{AX}$ foci in combination therapy-treated cells compared to monotherapy of ERX-11 or palbociclib or untreated control (Additional file 1: Figure S9).
ERX-11 and palbociclib treatment decrease the levels of E2F1, ERa, and ER-coregulators

Western blot analysis using two BCa models also confirmed the downregulation of ER $\alpha$ and E2F1 expression upon treatment with combination therapy compared to monotherapy (Fig. 7a). Further, western blot analysis revealed that the combination therapy reduced the levels of several oncogenic ER coregulators including SRC3, PELP1, and SRC1 (Fig. 7b). Moreover, western blot analyses confirmed the downregulation of DNA repair proteins identified in DIA analyses including FOXM1, PARP1, and NF-kB subunit p65 following combination 
therapy (Fig. 7c). Since global proteomic analysis results (Fig. 6) suggested the downregulation of pathways involved in protein translation, we confirmed whether ERX11 and palbociclib therapy alters the mTOR signaling axis. The results showed that ERX-11 and palbociclib therapy substantially reduced the expression of several components of mTOR signaling including mTOR, 4EBP1, and p70S6K compared to monotherapy (Fig. 7d). The RTqPCR analysis confirmed the significant downregulation of several genes regulated by the E2F1 and ER pathways by combination therapy (Fig. 7e). These data indicate that the downregulation of E2F1, ER $\alpha$, and ER binding proteins may contribute functionally to the alteration in the ER coregulator binding profile and consequently to ER activity. Taken together, these results along with unbiased global mass spectrometry-based analyses (Fig. 6) indicate that the effect of the combination therapy may be related to the dramatic decrease of the expression of a number of ER coregulators, ER, and E2F downstream signaling proteins (Additional file 1: Figure S10).

\section{Discussion}

The majority of breast cancer is ER+. However, both de novo and acquired therapy resistance limit the utility of ER-targeted therapy using AIs and AEs. Emerging evidence implicate ER signaling is intact in many therapy-resistant tumors, and ER interaction with critical coregulator proteins mediates ER signaling in these therapy-resistant tumors. While AIs and AEs may disrupt some of these ER-coregulator interactions, their ability to target these interactions is limited in therapy-resistant cells. Recently, we developed ERX-11, a small molecule that targets ER at a unique site, that functions as an ER-coregulator modulator and has good efficacy in treating therapy-resistant $\mathrm{BCa}$ [23]. Since extensive crosstalk occurs with cell cycle machinery and ER coregulators, we reasoned that combination treatments that block ER-coregulator signaling and CDK4/6 signaling may have better therapeutic utility. Using multiple in vitro cell-based assays, ex vivo PDE, xenograft-derived MT-ER explants, and

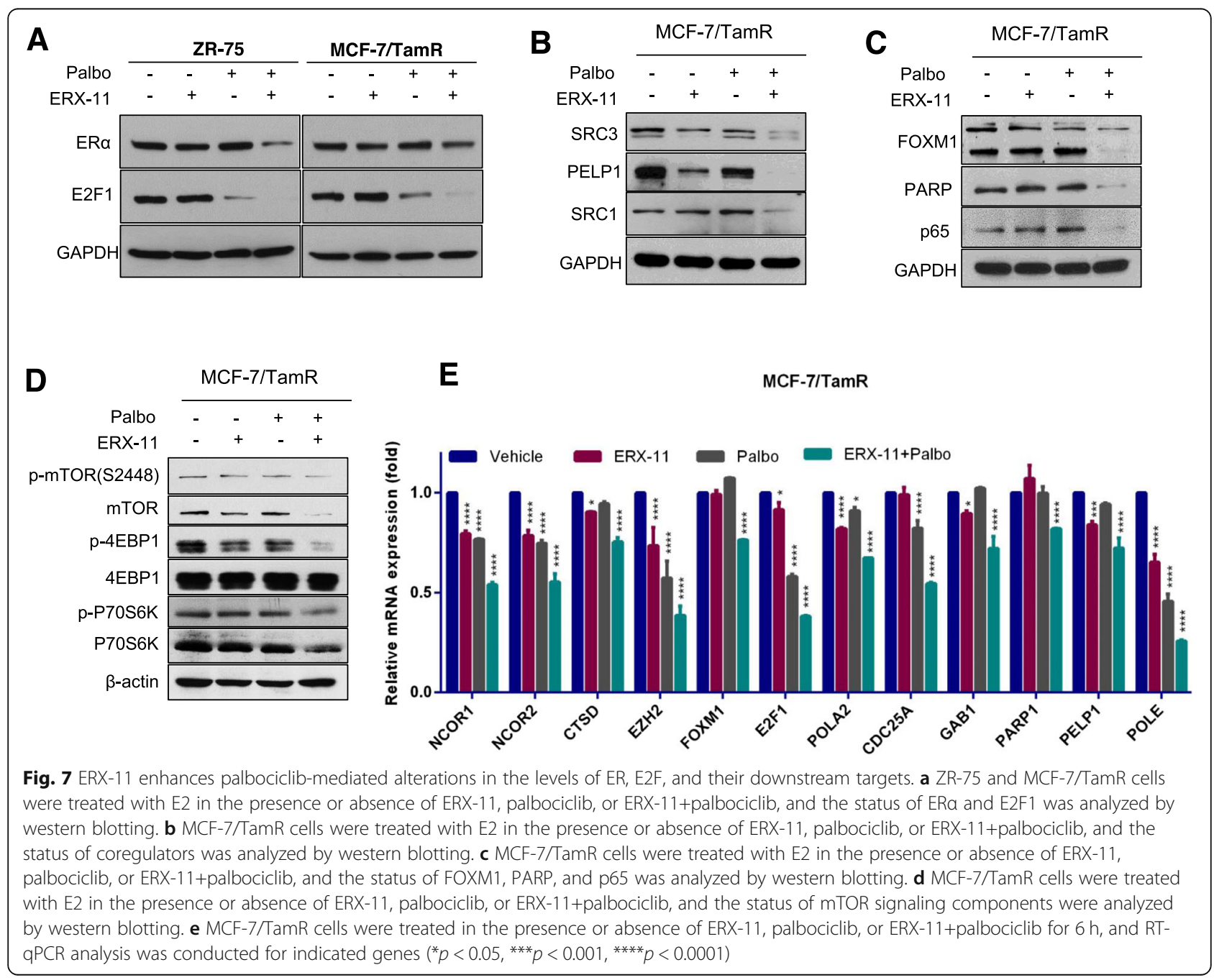


preclinical xenograft models of endocrine resistance, this study provided evidence that combination therapy of ERX-11 and palbociclib is very efficient in blocking the growth of therapy-resistant $\mathrm{BCa}$.

Approximately $70 \%$ of $\mathrm{BCa}$ are dependent on ER signaling for growth. ER signaling modulates multiple pathways including upregulation of cyclin D1 which upregulate CDK4/6 activity [35, 36]. Cyclin D1 amplification occurs in $58 \%$ of luminal B cancers and $29 \%$ of luminal A cancers [20]. Increased cyclin D1 potentiates ER transcription in an autocrine manner further potentiating ER-mediated cell proliferation. This scientific premise formed the basis for combining endocrine therapy with CDK4/6 inhibitors to overcome ER therapy resistance. Recently, the FDA approved CDK4/6 inhibitors for clinical use in the metastatic setting in combination with letrozole or with fulvestrant [37]. All three CDK4/6 inhibitors (palbociclib, ribociclib, abemaciclib) showed prolongation of progression-free survival (PFS) over ER targeted therapy. Unfortunately, most patients eventually acquire resistance to CDK4/6 inhibitors, and disease progression occurs. Preclinical studies identified several mechanisms that contribute to resistance including acquired mutation of RB1, amplification of cyclin E, amplification of CDK6 or suppression of CDK2 inhibitors (e.g., p27kip1 or p21cip1), and alterations in the $\mathrm{PI} 3 \mathrm{~K} / \mathrm{mTOR}$ pathway [38]. Therefore, combining CDK4/6 inhibitor therapy with an effective ER target therapy will further maximize CDK4/6 inhibitors utility. Our results support the utility of ERX-11 in extending the efficacy of CDK4/6 inhibitors (palbociclib, ribociclib, abemaciclib) for treating therapy-resistant BCa. Further, ERX-11 was also effective in reducing the growth of CDK4/6 inhibitorresistant models.

ER-coregulators play an important role in ER mediated cell proliferation. For example, SRC3 directly interacts with E2F1, is recruited to E2F target gene promoters, and stimulates the transcription of a subset of E2Fresponsive genes that are associated with the G1/S transition [39]. Knockdown of SRC2 or SRC3 coactivators decreases the E2-induced progression from G1 to $S$ [40]. ER coregulator, PELP1, is a novel substrate of CDKs, phosphorylated by the CDK4/cyclin D1, couples ER signaling to the E2F axis, and CDK phosphorylation plays a key role in the PELP1 oncogenic functions [18]. ERcoregulator CARM1 is required for the E2-mediated activation of E2F1 and for the induction of E2F1 target genes [41]. Since crosstalk occurs between ER and E2F1 signaling, blockage of ER-coregulator signaling further potentiates the inhibition of E2F signaling mediated by palbociclib. Our results showed that the combination of ERX-11 and palbociclib is more efficient in decreasing E2F1 protein levels compared to monotherapy of ERX11 or palbociclib. Further, our studies demonstrated a higher efficacy of combination therapy in altering both
ER and E2F signaling. These results suggest that the cotherapeutic targeting of ERX-11 with palbociclib is beneficial in treating therapy-resistant $\mathrm{BCa}$.

Alterations in the concentration or activity of selective coregulators enable ER signaling from AE-ER complexes, effectively converting the antagonist to an agonist [15, 16]. Over one third (38\%) of ER coregulators identified in BCa are over-expressed [4-6], such as SRC3 [7, 8], SRC2 [9], and PELP1 [10]. The unbiased IP mass spectrometry analyses suggest that ERX-11 and palbociclib combination is more efficient in blocking the interactions of ER with multiple coregulators. Several mechanisms might have contributed to the increased efficacy of ERX-11 and palbociclib combination in the disruption of the ER interactome. Since ER and its coregulator expression and functions are modulated throughout the cell cycle via phosphorylation by CDKs, CDK4/6 inhibition could indirectly affect ER interactions by altering ER phosphorylation, coregulator phosphorylation, and their expression by altering ER and E2F transcription factors. In support of this, RT-qPCR analyses showed that ERX-11 and palbociclib combination therapy is more efficient in suppressing key pathways including ER and E2F. Western blot analyses showed that combination therapy has the potential to reduce the expression levels of several coregulators. Thus, the more comprehensive disruption of ER coregulators by the combination of ERX-11 and CDK4/6 inhibitors represents an exciting approach in therapy-resistant $\mathrm{BCa}$.

Mutations in ER are rare in untreated patients. However, recent studies revealed that breast tumors acquire mutations in the ER ligand-binding domains (L536 N, Y537S, Y537N, and D538G) that facilitate the constitutive activity of these mutant ER (MT-ER) in the absence of ligand [29-32]. Tumors with MT-ER interact with oncogenic coregulators to drive ERdependent transcriptional programs and proliferation and are poorly responsive to AEs and AIs [17, 29-32]. Recent studies reported encouraging activity of fulvestrant and palbociclib combination therapy against MT-ER cancers; however, it also suggested that combination treatment with palbociclib and letrozole does not prevent the selection of ER mutations [42]. Therefore, endocrine agents that are effective against MTER will have more efficacy in treating advanced BCa. ERX-11 interacts directly with MT-ER and efficiently blocks their oncogenic signaling [23]. Our results showed that ERX-11 significantly enhanced the efficacy of palbociclib in reducing the growth of MT-ER expressing cells. Importantly, using ex vivo culture of xenograft-derived MT-ER tumor tissues, we demonstrated that ERX-11 and palbociclib combination therapy is effective in limiting the proliferation of ER-MTdriven tumors. 
E2F-mediated gene transcription plays a critical role in DNA damage response and repair [43]. CDK4/6 inhibitors are known to cause suppression of poly (ADP-ribose) polymerase 1 (PARP1) transcription [44], and PARP1 protein is involved in several mechanisms of DNA damage repair. In this study using DIA based whole-cell lysate mass spectrometry analysis, we found that ERX-11 and palbociclib combination therapy significantly downregulated the DNA damage response and repair pathways compared to monotherapy [45]. Independent biochemical assays also confirmed the downregulation of FOXM1 and PARP1 proteins. Since FOXM1 drives the transcription of genes for DNA damage sensors, mediators, signal transducers, and effectors [46], a decrease in FOXM1 reduces the DNA damage repair gene expression network and this might have contributed to enhanced sensitization of ERX11 and palbociclib combination therapy.

\section{Conclusions}

Our findings suggested that combination therapy of ERX-11 and CDK4/6 inhibitors may be a promising therapeutic strategy for therapy-resistant BCa. Mechanistic studies revealed that ERX-11 and CDK4/6 inhibitor combination therapy potency is mediated by (1) higher efficacy in altering both ER and E2F signaling, (2) more comprehensive disruption of ER coregulators, and (3) decreased DNA damage repair gene expression network. Since ERX-11 is well-tolerated with fewer side effects and has activity against $\mathrm{BCa}$ resistant to both endocrine therapy and CDK4/6 inhibitors, it can be readily extended to clinical use as a therapeutic to enhance the utility of CDK4/6 inhibitors.

\section{Supplementary information}

Supplementary information accompanies this paper at https://doi.org/10. 1186/s13058-019-1227-8.

Additional file 1: Figure S1. (A) MCF-7 or (B) T-47D cells were stimulated with E2 $\left(10^{-8} \mathrm{M}\right)$ for 3 days in the presence or absence of $1 \mu \mathrm{M}$ of ERX-11 or cisplatin or paclitaxel or gemcitabine or in combination and the cell viability was measured by Cell Titre-Glo Luminescent assay. Figure S2. ZR-75, T-47D, MCF-7/TamR, ZR-75-ESR1-MT-Y537S and ZR-75ESR1-MT-D538G cells were stimulated with E2 $\left(10^{-8} \mathrm{M}\right)$ for 7 days in the presence or absence of ERX-11 $(0.5 \mu \mathrm{M})$ or palbociclib $(0.5 \mu \mathrm{M})$ or in combination with indicated concentrations of ERX-11 and the cell viability was measured by MTT assay. Figure S3. Equal number of ZR-75, ZR-75ESR1-MT-Y537S and ZR-75-ESR1-MT-D538G cells were plated and treated with ERX-11 (500nM) or palbociclib (50 nM) or abemaciclib (50nM) or ribociclib (50nM) or combination and clonogenic (survival) assays were performed after 14 days. Figure S4. (A) Parental MCF-7 or ribociclib resistant MCF-7/RR cells were stimulated with E2 $\left(10^{-8 \mathrm{M}}\right)$ for 5 days in the presence or absence of ERX-11 $(1,5,10 \mu \mathrm{M})$ or ribociclib $(1 \mu \mathrm{M})$ or in combination and the cell viability was measured by Cell Titer-Glo Luminescent assay. (B) MCF-7 or (C) MCF-7/RR cells were treated with E2 $\left(10^{-8} \mathrm{M}\right)$ for 5 days in the presence or absence of ERX-11 $(1,2,5 \mu \mathrm{M})$ or $\mathrm{ICl}$ $(0.2,0.4,1 \mu \mathrm{M})$. Figure S10. Schematic representation of model for mechanisms of ERX-11+palbociclib therapy.

\section{Abbreviations}

AE: Anti-estrogen; Al: Aromatase inhibitor; CDK: Cell cycle-dependent kinase; DIA: Data-independent acquisition; E2: Estrogen; ER: Estrogen receptor; ERX11: Estrogen receptor coregulator binding modulator -11; ESR1: Estrogen receptor alpha; IHC: Immunohistochemistry; MCF-7/LTLTca: MCF7 cells resistant to letrozole; MCF-7/RR: MCF7 cells: resistant to ribociclib; MCF7Tam: MCF7 cells resistant to tamoxifen; MT-ER: Estrogen receptor with mutation; PDE: Patient-derived explants; PELP1: Proline, glutamic acid, and leucine-rich protein-1; PI3K: Phosphotidyl inositol 3-kinase; RT-qPCR: Real-time quantitative PCR; SERD: Selective estrogen receptor degrader; SRC: Steroid receptor coregulator; XDE: Xenograft-derived explants

\section{Acknowledgements}

This manuscript is dedicated to Dr. Rui Li. She was actively involved in these projects until her untimely demise from cancer. We are grateful for the expert technical assistance of Sammy Pardo and Dana Molleur.

\section{Authors' contributions}

RKV, RRT, GVR, J-MA, GRS, SV, SM, STW, CA, and MR designed the experiments and interpreted the results. SV, SM, YL, GRS, XL, CG, TKL, MZ, EBB, KK, $P A, M L$, and UPP, conducted the experiments. RKV, GRS, SV, SM, GVR, and JMA wrote the manuscript. All authors read and approved the final manuscript.

\section{Funding}

This study was supported by the NIH CA223828-01 (RKV, GVR, J-MA), NCI Cancer Center Support Grant P30CA054174-17 (RV, STW), Voelcker Fund Young Investigator Grant (GRS), NIH grant 1R01CA179120-01 (RKV and MR), Bank of America foundation (SV), and Welch Foundation AT-1595 (J-MA). Mass spectrometry analyses were conducted at the UTHSA Institutional Mass Spectrometry Laboratory supported in part by UTH SA and the University of Texas Proteomics Core Network (STW) for the purchase of the Lumos mass spectrometer.

\section{Availability of data and materials}

All data generated for this study are included within this article and in the supplementary information.

\section{Ethics approval and consent to participate}

All animal experiments were performed after obtaining UTHSA IACUC approval using the methods in the approved protocol. For explant experiments, deidentified patient tumors were obtained from the UTSW Tissue Repository after institutional review board approval (STU-032011-187) [47].

\section{Consent for publication}

Not applicable

\section{Competing interests}

J-MA, GVR, and RV are joint holders on issued and pending patents on ERX11. CA serves or has served in an advisory role to Novartis, Merck, Lilly, Radius, Sanofi, Daiichi Sankyo, H3 Biomedicine, Puma, TAlHO Oncology, Symphogen, Bayer, Athenex, G1 Therapeutics, Petra Pharma and Celgene. He also has stock options in Provista and Y-TRAP. He has or has had grant support from Lilly, Pfizer, Bayer, Takeda and Radius. He also serves in the Scientific Advisory Board of the Komen Foundation. The other authors declare that they have no competing interests.

\section{Author details}

${ }^{1}$ Department of Obstetrics and Gynecology, University of Texas Health, San Antonio, TX 78229, USA. ²Departments of Urology and Pharmacology, University of Texas Southwestern Medical Center at Dallas, Dallas, TX 75390, USA. ${ }^{3}$ CDP Program, University of Texas Health Cancer Center, San Antonio, TX 78229, USA. ${ }^{4}$ Department of Chemistry and Biochemistry, University of Texas at Dallas, Richardson, TX 75080, USA. ${ }^{5}$ Simmons Cancer Center, University of Texas Southwestern Medical Center at Dallas, Dallas, TX 75390, USA. ${ }^{6}$ Department of Radiation Oncology, University of Texas Southwestern Medical Center at Dallas, Dallas, TX 75390, USA. ${ }^{7}$ Department of Cell Systems and Anatomy, University of Texas Health, San Antonio, TX 78229, USA. ${ }^{8}$ Department of Biochemistry and Structural Biology, University of Texas Health, San Antonio, TX 78229, USA. 
Received: 10 June 2019 Accepted: 13 November 2019 Published online: 26 December 2019

\section{References}

1. Turner NC, Neven P, Loibl S, Andre F. Advances in the treatment of advanced oestrogen-receptor-positive breast cancer. Lancet (London, England). 2017;389(10087):2403-2414.

2. Musgrove EA, Sutherland RL. Biological determinants of endocrine resistance in breast cancer. Nat Rev Cancer. 2009:9(9):631-43.

3. Dasgupta S, O'Malley BW. Transcriptional coregulators: emerging roles of SRC family of coactivators in disease pathology. J Mol Endocrinol. 2014; 53(2):R47-59.

4. Lonard DM, Lanz RB, O'Malley BW. Nuclear receptor coregulators and human disease. Endocr Rev. 2007;28(5):575-87.

5. Lonard DM, O'Malley BW. Nuclear receptor coregulators: modulators of pathology and therapeutic targets. Nat Rev Endocrinol. 2012;10:598-604.

6. Cortez V, Samayoa C, Zamora A, Martinez L, Tekmal RR, Vadlamudi RK. PELP1 overexpression in the mouse mammary gland results in the development of hyperplasia and carcinoma. Cancer Res. 2014;74(24):7395-405.

7. List HJ, Reiter R, Singh B, Wellstein A, Riegel AT. Expression of the nuclear coactivator AIB1 in normal and malignant breast tissue. Breast Cancer Res Treat. 2001;68(1):trtr21-8.

8. Azorsa DO, Cunliffe HE, Meltzer PS. Association of steroid receptor coactivator AIB1 with estrogen receptor-alpha in breast cancer cells. Breast Cancer Res Treat. 2001;70(2):89-101.

9. Kurebayashi J, Otsuki T, Kunisue H, Tanaka K, Yamamoto S, Sonoo H. Expression levels of estrogen receptor-alpha, estrogen receptor-beta, coactivators, and corepressors in breast cancer. Clin Cancer Res. 2000;6(2):512-8.

10. Habashy HO, Powe DG, Rakha EA, Ball G, Macmillan RD, Green AR, et al. The prognostic significance of PELP1 expression in invasive breast cancer with emphasis on the ER-positive luminal-like subtype. Breast Cancer Res Treat. 2010;120(3):603-12.

11. Popov VM, Zhou J, Shirley LA, Quong J, Yeow WS, Wright JA, et al. The cell fate determination factor $\mathrm{DACH} 1$ is expressed in estrogen receptor-alphapositive breast cancer and represses estrogen receptor-alpha signaling. Cancer Res. 2009;69(14):5752-60.

12. Shou J, Massarweh S, Osborne CK, Wakeling AE, Ali S, Weiss H, et al. Mechanisms of tamoxifen resistance: increased estrogen receptor-HER2/neu cross-talk in ER/ HER2-positive breast cancer. J Natl Cancer Inst. 2004:96(12):926-35.

13. Burandt $E$, Jens $G$, Holst F, Janicke F, Muller V, Quaas A, et al. Prognostic relevance of $\mathrm{AIB1}(\mathrm{NCOA} 3)$ amplification and overexpression in breast cancer. Breast Cancer Res Treat. 2013;137(3):745-53.

14. Girard BJ, Daniel AR, Lange CA, Ostrander JH. PELP1: a review of PELP1 interactions, signaling, and biology. Mol Cell Endocrinol. 2014;382(1):642-51.

15. O'Hara J, Vareslija D, McBryan J, Bane F, Tibbitts P, Byrne C, et al. AIB1: ERalpha transcriptional activity is selectively enhanced in aromatase inhibitor-resistant breast cancer cells. Clin Cancer Res. 2012;18(12):3305-15.

16. Kurebayashi J. Endocrine-resistant breast cancer: underlying mechanisms and strategies for overcoming resistance. Breast Cancer. 2003;10(2):112-9.

17. Toy W, Weir H, Razavi P, Lawson M, Goeppert AU, Mazzola AM, et al. Activating ESR1 mutations differentially affect the efficacy of ER antagonists. Cancer Discov. 2016.

18. Nair BC, Nair SS, Chakravarty D, Challa R, Manavathi B, Yew PR, et al. Cyclindependent kinase-mediated phosphorylation plays a critical role in the oncogenic functions of PELP1. Cancer Res. 2010;70(18):7166-75.

19. Foster JS, Henley DC, Bukovsky A, Seth P, Wimalasena J. Multifaceted regulation of cell cycle progression by estrogen: regulation of $\mathrm{Cdk}$ inhibitors and Cdc25A independent of cyclin D1-Cdk4 function. Mol Cell Biol. 2001; 21(3):794-810

20. Cancer Genome Atlas N. Comprehensive molecular portraits of human breast tumours. Nature. 2012:490(7418):61-70.

21. Shah AN, Cristofanilli M. The growing role of CDK4/6 inhibitors in treating hormone receptor-positive advanced breast cancer. Curr Treat Options in Oncol. 2017:18(1):6.

22. Schmidt M, Sebastian M. Palbociclib-the first of a new class of cell cycle inhibitors. Recent Results Cancer Res. 2018:211:153-75.

23. Raj GV, Sareddy GR, Ma S, Lee TK, Viswanadhapalli S, Li R, et al. Estrogen receptor coregulator binding modulators (ERXs) effectively target estrogen receptor positive human breast cancers. Elife. 2017;6:e26857.

24. Jansen VM, Bhola NE, Bauer JA, Formisano L, Lee KM, Hutchinson KE, et al. Kinome-wide RNA interference screen reveals a role for PDK1 in acquired resistance to CDK4/6 inhibition in ER-positive breast cancer. Cancer Res. 2017;77(9):2488-99.

25. Nabha SM, Glaros S, Hong M, Lykkesfeldt AE, Schiff R, Osborne K, et al. Upregulation of PKC-delta contributes to antiestrogen resistance in mammary tumor cells. Oncogene. 2005;24(19):3166-76.

26. Macedo LF, Sabnis G, Brodie A. Preclinical modeling of endocrine response and resistance: focus on aromatase inhibitors. Cancer. 2008;112(3 Suppl):679-88.

27. Cortez V, Mann M, Tekmal S, Suzuki T, Miyata N, Rodriguez-Aguayo C, et al. Targeting PELP1-KDM1 axis as a potential therapeutic strategy for breast cancer. Breast Cancer Res. 2012;14(4):R108.

28. Chou TC. Drug combination studies and their synergy quantification using the Chou-Talalay method. Cancer Res. 2010;70(2):440-6.

29. Toy W, Shen Y, Won H, Green B, Sakr RA, Will M, et al. ESR1 ligand-binding domain mutations in hormone-resistant breast cancer. Nat Genet. 2013; 45(12):1439-45.

30. Robinson DR, Wu YM, Vats P, Su F, Lonigro RJ, Cao X, et al. Activating ESR1 mutations in hormone-resistant metastatic breast cancer. Nat Genet. 2013; 45(12):1446-51.

31. Jeselsohn R, Yelensky R, Buchwalter G, Frampton G, Meric-Bernstam F, Gonzalez-Angulo AM, et al. Emergence of constitutively active estrogen receptor-alpha mutations in pretreated advanced estrogen receptor-positive breast cancer. Clin Cancer Res. 2014;20(7):1757-67.

32. Merenbakh-Lamin K, Ben-Baruch N, Yeheskel A, Dvir A, Soussan-Gutman L, Jeselsohn $\mathrm{R}$, et al. D538G mutation in estrogen receptor-alpha: a novel mechanism for acquired endocrine resistance in breast cancer. Cancer Res. 2013;73(23):6856-64.

33. Dean JL, McClendon AK, Hickey TE, Butler LM, Tilley WD, Witkiewicz AK, et al. Therapeutic response to CDK4/6 inhibition in breast cancer defined by ex vivo analyses of human tumors. Cell Cycle. 2012;11(14):2756-61.

34. Schiewer MJ, Goodwin JF, Han S, Brenner JC, Augello MA, Dean JL, et al. Dual roles of PARP-1 promote cancer growth and progression. Cancer Discov. 2012;2(12):1134-49.

35. Lundgren K, Brown M, Pineda S, Cuzick J, Salter J, Zabaglo L, et al. Effects of cyclin D1 gene amplification and protein expression on time to recurrence in postmenopausal breast cancer patients treated with anastrozole or tamoxifen: a TransATAC study. Breast Cancer Res. 2012;14(2):R57.

36. Finn RS, Aleshin A, Slamon DJ. Targeting the cyclin-dependent kinases (CDK) 4/6 in estrogen receptor-positive breast cancers. Breast Cancer Res. 2016;18(1):17

37. Preusser M, De Mattos-Arruda L, Thill M, Criscitiello C, Bartsch R, Ruhstaller T, et al. CDK4/6 inhibitors in the treatment of patients with breast cancer: summary of a multidisciplinary round-table discussion. ESMO Open. 2018; 3(5):e000368.

38. Herrera-Abreu MT, Palafox M, Asghar U, Rivas MA, Cutts RJ, Garcia-Murillas I, et al. Early adaptation and acquired resistance to CDK4/6 inhibition in estrogen receptor-positive breast cancer. Cancer Res. 2016;76(8):2301-13.

39. Louie MC, Zou JX, Rabinovich A, Chen HW. ACTR/AIB1 functions as an E2F1 coactivator to promote breast cancer cell proliferation and antiestrogen resistance. Mol Cell Biol. 2004;24(12):5157-71.

40. Karmakar S, Foster EA, Smith CL. Unique roles of p160 coactivators for regulation of breast cancer cell proliferation and estrogen receptor-alpha transcriptional activity. Endocrinology. 2009;150(4):1588-96.

41. Frietze S, Lupien M, Silver PA, Brown M. CARM1 regulates estrogenstimulated breast cancer growth through up-regulation of E2F1. Cancer Res. 2008;68(1):301-6.

42. Fribbens C, O'Leary B, Kilburn L, Hrebien S, Garcia-Murillas I, Beaney M, et al. Plasma ESR1 mutations and the treatment of estrogen receptor-positive advanced breast cancer. J Clin Oncol. 2016;34(25):2961-8.

43. Biswas AK, Mitchell DL, Johnson DG. E2F1 responds to ultraviolet radiation by directly stimulating DNA repair and suppressing carcinogenesis. Cance Res. 2014;74(12):3369-77.

44. Tempka D, Tokarz P, Chmielewska K, Kluska M, Pietrzak J, Rygielska Z, et al. Downregulation of PARP1 transcription by CDK4/6 inhibitors sensitizes human lung cancer cells to anticancer drug-induced death by impairing OGG1-dependent base excision repair. Redox Biol. 2018;15:316-26.

45. Dean JL, McClendon AK, Knudsen ES. Modification of the DNA damage response by therapeutic CDK4/6 inhibition. J Biol Chem. 2012;287(34): 29075-87.

46. Zona S, Bella L, Burton MJ, Nestal de Moraes G, Lam EW. FOXM1: an emerging master regulator of DNA damage response and genotoxic agent resistance. Biochim Biophys Acta. 2014;1839(11):1316-22. 
47. Mohammed H, Russell IA, Stark R, Rueda OM, Hickey TE, Tarulli GA, et al. Progesterone receptor modulates ERalpha action in breast cancer. Nature. 2015;523(7560):313-7.

\section{Publisher's Note}

Springer Nature remains neutral with regard to jurisdictional claims in published maps and institutional affiliations.

Ready to submit your research? Choose BMC and benefit from:

- fast, convenient online submission

- thorough peer review by experienced researchers in your field

- rapid publication on acceptance

- support for research data, including large and complex data types

- gold Open Access which fosters wider collaboration and increased citations

- maximum visibility for your research: over $100 \mathrm{M}$ website views per year

At $\mathrm{BMC}$, research is always in progress. 